\title{
GAME THEORETIC ANALYSIS FOR AN OPTIMAL STOPPING PROBLEM BY MEANS OF MOMENTS OF A DISTRIBUTION FUNCTION
}

\author{
Jun-ichi Nakagami \\ Chiba University
}

(Received June 4, 1990; Revised September 6, 1991)

\begin{abstract}
Let $X_{1}, X_{2}, \cdots, X_{n}, \cdots$ be mutually independent random variables with a common cdf $F$, which is unknown but belongs to some class $\mathcal{F}$ of cdf's. The class $\mathcal{F}=\mathcal{F}\left(\mu, \sigma^{2}, M\right)$ is the set of all cdf's whose mean, variance and domain are $-\infty<\mu<\infty, 0<\sigma^{2}<\infty$, and $[\mu-M, \mu+M]$ respectively. It is assumed that they are known. Under an observation cost $c, 0<c<\infty$, we consider a stopping problem $\phi(x, F)$ as a two-person zero-sum game in which the player 1 decides his stopping set $\{X>x\}, x \in R$, and the player 2 chooses her cdf $F$ in $\mathcal{F}$. We analyze the upper bound problem $\phi^{u}=\sup _{x \in R} \sup _{F \in \mathcal{F}} \phi(x, F)$ and the game problem $\phi^{s}=$ value $_{x \in R, F \in \mathcal{F}} \phi(x, F)$ to derive a simple and meaningful solution with the parameters $c, \mu, \sigma$ and $M$.
\end{abstract}

\section{Introduction}

\subsection{Preliminaries}

Let $X_{1}, X_{2}, \cdots, X_{n}, \cdots$ be mutually independent and identically distributed random variables with a common cdf $F(t)=P\{X \leq t\}$ such that $E\left[X^{+}\right]=\int_{R_{+}} t d F(t)<\infty$, where $R=$ $(-\infty, \infty), R_{+}=[0, \infty)$. A positive observation cost $c\left(\in R_{++}=(0, \infty)\right)$ is incurred to the observation of each $X_{n}, n \geq 1$. If the observation process is stopped after $X_{n}$ is observed, a reward $X_{n}-n c$ is received.

When the cdf $F$ is known precisely, an optimal stopping problem is to find a stopping time $N$ which maximizes the expected reward $E\left[X_{N}-c N\right]$. An integer valued random variable $N$ is called a stopping time if, for each $n$, the event $\{N=n\}$ is independent of $X_{n+1}, X_{n+2}, \cdots$. This is a typical example of the stationary stopping problem (cf. Chow, Robbins and Siegmund $[1, \mathrm{p} .56]$ ), in which the optimal stopping time $N$ is necessarily of the form: to stop at $N=\min \left\{n \mid X_{n} \in S\right\}$ for some stopping set $S \subset R$, and $S$ is stationary and of a control-limit-type $\{X \geq x\}$ or $\{X>x\}$ for some $x \in R$, where $x$ is called a stopping level. For this, we define that a stopping level $x$ (or $x-0$ ) means a stopping set $\{X>x\}$ (or $\{X \geq x\}$ ) respectively.

For any stopping level $x$ and for any cdf $F$, we define an expected reward $\phi(x, F)=E\left[X_{N}-c N\right]$ of the stopping problem by

$$
\phi(x, F)=\frac{\int_{(x, \infty)} t d F(t)-c}{\bar{F}(x)}=x+\frac{\int_{(x, \infty)}(t-x) d F(t)-c}{\bar{F}(x)},
$$

where $\bar{F}(x)=1-F(x)$. Note that $\bar{F}(x) \rightarrow 0$ and $\phi \rightarrow-\infty$ as $x \rightarrow \infty$ and that $\bar{F}(x) \rightarrow 1$ and $\phi \rightarrow \mu_{F}-c$ as $x \rightarrow-\infty$ where $\mu_{F}=E[X]=\int_{R} t d F(t)$. It means that to continue the observation process indefinitely gives us the reward $-\infty$ and to stop the process immediately gives us the reward $\mu_{F}-c$. 
By the assumption $E\left[X^{+}\right]<\infty$, using integration by parts, we have

$$
\int_{(x, \infty)}(t-x) d F(t)=-\int_{(x, \infty)}(t-x) d \bar{F}(t)=\int_{x}^{\infty} \bar{F}(t) d t=\int_{[x, \infty)}(t-x) d F(t),
$$

which is continuous in $x$. To handle this expectation, it is convenient to define $T_{F}(x)$,

$$
T_{F}(x)=\int_{x}^{\infty}(t-x) d F(t)=\int_{x}^{\infty} \bar{F}(t) d t .
$$

It has some useful properties, which can be obtained by the elementary analytical technique. Then we give Lemma 1 without proof.

Lemma 1. $T_{F}(x)$ is continuous, non-negative, convex and non-increasing function of $x$. It satisfies that $T_{F}(x) \geq\left(\mu_{F}-x\right)^{+}$for any $x \in R$ and that $T_{F}(x) \rightarrow+\infty$ as $x \rightarrow-\infty$ and $T_{F}(x) \rightarrow 0$ as $x \rightarrow+\infty$. $T_{F}$ has a derivative a.e.. Moreover, if $T_{F}(x)$ is positive at any point $x$, it is strictly decreasing at $x$.

Now, redefining the expected reward $\phi(x, F)$ by $\left(1.1^{\prime}\right)$ for any stopping level $x$ and for any cdf $F$, we will have the optimal expected reward $\phi^{\circ}(F)$ for any cdf $F$ :

$$
\begin{gathered}
\phi(x, F)=x+\frac{T_{F}(x)-c}{\bar{F}(x)} . \\
\phi^{\circ}(F) \stackrel{\text { def }}{=} \sup _{x \in R} \phi(x, F) . \\
\frac{d \phi(x, F)}{d F(x)}=\frac{T_{F}(x)-c}{\bar{F}^{2}(x)} .
\end{gathered}
$$

The right hand side of (1.4) changes the sign from + to - at most one time as $x$ goes from $-\infty$ to $+\infty$. From Lemma 1, the equation $T_{F}(x)=c$ for any fixed $c(c>0)$ has a unique solution $x^{\circ}(F) \stackrel{\text { def }}{=}\left(T_{F}\right)^{-1}(c)$, so that the set of optimal stopping levels $\mathbf{x}^{\circ}(F)$ (which must contain the point $\left.x^{\circ}(F)\right)$ of $(1.3)$ is given by

$$
\mathbf{x}^{o}(F)=\left\{x \mid F(x)=F\left(x^{o}(F)\right)\right\} .
$$

Since the $\operatorname{cdf} F$ is right-continuous, this set is an interval of the form $[a, b)$. If the $\operatorname{cdf} F$ has a positive density in the neighborhood of $x^{\circ}(F)$, the set $\mathbf{x}^{\circ}(F)$ contains only one point $x^{\circ}(F)$ i.e., $\mathbf{x}^{o}(F)=\left\{x^{o}(F)\right\}$.

We have the optimal expected reward $\phi^{\circ}(F)$,

$$
\phi^{\circ}(F)=x^{\circ}(F)=\phi\left(\mathbf{x}^{\circ}(F), F\right),
$$

where $\phi(A, F)$ means $\phi(x, F)$ for any $x$ in a set $A$.

Let $a=\min \left\{x \mid x \in \mathbf{x}^{\circ}(F)\right\}$ and $b=\sup \left\{x \mid x \in \mathbf{x}^{\circ}(F)\right\}$, then the set $\mathbf{x}^{\circ}(F)$ of (1.5) is represented as the interval $[a, b)$. Since $F$ has no probability mass in $(a, b)$

$$
\bar{F}(a)=P\{X>a\}=P\{X \geq b\}=\bar{F}(b-0) \stackrel{\text { def }}{=} \lim _{x \uparrow b} \bar{F}(x), \int_{(a, \infty)} t d F(t)=\int_{[b, \infty)} t d F(t),
$$


and by (1.1) it holds that,

$$
x^{o}(F)=\phi(a, F)=\lim _{x \uparrow b} \phi(x, F) \stackrel{\text { def }}{=} \phi(b-0, F) .
$$

Then, we have Lemma 2.

Lemma 2. For any given $c d f F$, the following stopping sets or stopping levels $(i)(i i)(i i i)$ are optimal, and the optimal expected reward is given by $\left(1.3^{\prime \prime}\right)$;

(i) the set $\{X>a\}$ or level $a$ where $a=\min \left\{x \mid x \in \mathbf{x}^{o}(F)\right\}$,

(ii) the set $\{X \geq b\}$ or level $b-0$ where $b=\sup \left\{x \mid x \in \mathbf{x}^{\circ}(F)\right\}$,

(iii) the set $\{X>x\}(\{X \geq x\})$ or level $x(x-0)$ where $\forall x \in(a, b)$.

\subsection{Statement of the Problem}

The underlying premise is that some information is available about certain moments of an unknown cdf $F$. This determines some class $\mathcal{F}$ of cdf's. This limited information is to be used in order to obtain an upper (or/and lower) bound for our stopping problem $\phi(x, F)$ of $\left(1.1^{\prime}\right)$. In this paper, we consider the classes $\mathcal{F}$ of all cdf's $F$ which are unknown except for their first two moments and domain.

First, we shall consider the maximal bound $\phi^{u}$ for $\phi(x, F)$ on $R \times \mathcal{F}$

$$
\phi^{u}=\sup _{x \in R} \sup _{F \in \mathcal{F}} \phi(x, F)=\sup _{F \in \mathcal{F}} \phi^{o}(F)=\phi\left(x^{o}\left(F^{u}\right), F^{u}\right)=\phi\left(x^{u}, F^{u}\right),
$$

where $\left(x^{u}, F^{u}\right)$ is a joint maximizing point of $\phi(x, F)$.

Second, we shall consider $\phi(x, F)$ as a two-person zero-sum game in which the player 1 (gambler) decides his level $x$ in $R$ and the player 2 (nature) chooses her cdf $F$ in $\mathcal{F}$, before the observation of $\left\{X_{n} ; n \geq 1\right\}$. Then the minimax value $\phi^{*}$ and the maximin value $\phi_{*}$ on $R \times \mathcal{F}$,

$$
\begin{gathered}
\phi^{*}=\inf _{F \in \mathcal{F}} \sup _{x \in R} \phi(x, F)=\inf _{F \in \mathcal{F}} \phi^{\circ}(F)=\phi\left(x^{o}\left(F^{*}\right), F^{*}\right)=\phi\left(x^{*}, F^{*}\right), \\
\phi_{*}=\sup _{x \in R} \inf _{F \in \mathcal{F}} \phi(x, F)=\phi\left(x_{*}, F_{*}\right),
\end{gathered}
$$

and the saddle value $\phi^{s}$, the saddle point $\left(x^{s}, F^{s}\right)$ in $R \times \mathcal{F}$,

$$
\phi^{s}=\operatorname{value}_{x \in R, F \in \mathcal{F}} \phi(x, F)=\phi\left(x^{s}, F^{s}\right),
$$

will be derived for the following two classes $\mathcal{F}\left(\mu, \sigma^{2}\right)$ and $\mathcal{F}\left(\mu, \sigma^{2}, M\right)$ of cdf's.

The class $\mathcal{F}\left(\mu, \sigma^{2}, M\right)$ is the set of cdf's whose mean $\mu$, variance $\sigma^{2}$ and domain $[\mu-M, \mu+M]$ are assumed to be known.

$$
\begin{gathered}
\mathcal{F}\left(\mu, \sigma^{2}, M\right)=\left\{F \mid \int_{A} d F(t)=1, \int_{A} t d F(t)=\mu\right. \\
\left.\int_{A} t^{2} d F(t)=\mu^{2}+\sigma^{2} \text { where } A=[\mu-M, \mu+M], M \geq \sigma\right\}
\end{gathered}
$$

The class $\mathcal{F}\left(\mu, \sigma^{2}\right)$ is $\mathcal{F}\left(\mu, \sigma^{2}, M\right)$ where $M$ is arbitrary in $[\sigma, \infty)$, and $\mathcal{F}(\mu)$ is $\mathcal{F}\left(\mu, \sigma^{2}\right)$ where $\sigma^{2}$ is arbitrary in $R_{++}$.

The organization of this paper is as follows. In Section 2, we study some basic results for our stopping problem $\phi(x, F)$ and $T_{F}(x)$ and properties of cdf's $F$ in the class $\mathcal{F}$, which play fundamental roles in all that follows. Section 3 is a discussion about the existence and the 
derivation of the maximizing point and the saddle point for $\phi(x, F)$ in the class $\mathcal{F}=\mathcal{F}\left(\mu, \sigma^{2}\right)$. In fact, the class $\mathcal{F}$ is so rich for the player 2 that the player 1 must stop immediately and may receive the reward of $\mu-c$ as the saddle value. In this case, the information of the value $\sigma^{2}$ is useless for the player 1 .

To remove the non-interesting feature appearing in the game theoretic treatment in Section 3, we shall continue the discussion about the more interesting class $\mathcal{F}=\mathcal{F}\left(\mu, \sigma^{2}, M\right)$ in Section 4 . In this case, the information of the value $\sigma^{2}$ and $M$ is useful for the player 1 . The player 1 can receive the reward of $\mu+\left(\sigma^{2} / M-c\right)^{+}-c$ as the saddle value which is greater than $\mu-c$ in Section 3 .

\section{Some Fundamental Lemmas}

The general theory of a duality theorem is presented in the textbook by Ekeland and Teman [2, Chapter 4$]$. It is hardly difficult to obtain the condition of the existence of a saddle point for our stopping problem $\phi(x, F)$. For an inventory problem where this condition is satisfied, we have derived a saddle point [4]. The following two propositions are used to check a candidate for a saddle point of $\phi(x, F)$ on $R \times \mathcal{F}$.

Proposition 1. [2, p.166] If $\phi$ is a function defined on $R \times \mathcal{F}$ with real values, then

$$
\phi_{*}=\sup _{x \in R} \inf _{F \in \mathcal{F}} \phi(x, F) \leq \phi^{*}=\inf _{F \in \mathcal{F}} \sup _{x \in R} \phi(x, F) .
$$

Proposition 2. [2, p.168] If there exist $x^{s} \in R, F^{s} \in \mathcal{F}$ and a real value $\phi^{s}$ such that

$$
\begin{aligned}
& \phi\left(x^{s}, F\right) \geq \phi^{s} \text { for any } F \in \mathcal{F}, \\
& \phi\left(x, F^{s}\right) \leq \phi^{s} \text { for any } x \in R,
\end{aligned}
$$

then $\left(x^{s}, F^{s}\right)$ is a saddle point of $\phi$ and it holds that

$$
\phi^{s}=\text { value }_{x \in R, F \in \mathcal{F}} \phi(x, F)=\sup _{x \in R} \inf _{F \in \mathcal{F}} \phi(x, F)=\inf _{F \in \mathcal{F}} \sup _{x \in R} \phi(x, F) .
$$

Moreover, the set of saddle points of $\phi$ is of the form $\left(\mathbf{x}^{s}, \mathcal{F}^{s}\right)$, where $\mathbf{x}^{s} \subset R$ and $\mathcal{F}^{s} \subset \mathcal{F}$.

Before beginning our discussion, we shall establish some basic results for $\phi(x, F), T_{F}(x)$ and the class $\mathcal{F}$.

Let a random variable $X$ has a mean $\mu$ with a cdf $F_{\mu}(t)$, then the new random variable $X-\mu$ has the mean 0 with the cdf $F_{0}(t)=F_{\mu}(t+\mu)$. The following Lemma 3 follows immediately from the definition (1.1) of $\phi(x, F)$.

\section{Lemma 3.}

$$
\phi\left(x, F_{\mu}\right)=\mu+\phi\left(x-\mu, F_{0}\right) \text { for any } x \in R .
$$

Therefore, we may assume without loss of generality that all the cdf's in $F$ have the mean 0 . So that, we shall analyze the stopping problem in only two classes $\mathcal{F}\left(0, \sigma^{2}\right)$ and $\mathcal{F}\left(0, \sigma^{2}, M\right)$.

Lemma 4. For cdf's $F_{i}$ and non-negative numbers $\lambda_{i}, i=1,2, \cdots, n$, such that $\sum_{i=1}^{n} \lambda_{i}=1$, let $F=\sum_{i=1}^{n} \lambda_{i} F_{i}$. Then

$$
\phi(x, F)=\sum_{j=1}^{n} \lambda_{j}(x) \phi\left(x, F_{j}\right) \text { for any } x \in R, \text { where } \lambda_{j}(x)=\frac{\lambda_{j} \bar{F}_{j}(x)}{\sum_{i=1}^{n} \lambda_{i} \bar{F}_{i}(x)} .
$$


Proof. It follows at once from the definition (1.1) of $\phi$.

Note, from Lemma 4, that we find $\phi(x, F)$ is not necessarily convex in $F$ for any fixed $x \in R$, and that the well-known sufficient condition for the existence of a saddle point is not satisfied.

Let $G_{n}$ be a discrete cdf which has $n$ probability masses $p_{i}, p_{i}>0$, at $n$ points $t_{i}, i=1,2, \cdots, n$, respectively $\left(\sum_{i=1}^{n} p_{i}=1\right)$, i.e., it is represented as

$$
G_{n}(t)=\left(<t_{1}, \cdots, t_{n}><p_{1}, \cdots, p_{n}>\right)
$$

and $\mathcal{G}_{n}\left(\mu, \sigma^{2}\right)$ be all discrete cdf's $G_{n}$ in $\mathcal{F}\left(\mu, \sigma^{2}\right)$. Let

$$
G_{2}(t ; q)=\left(<-\frac{\sigma}{q}, \sigma q><\frac{q^{2}}{1+q^{2}}, \frac{1}{1+q^{2}}>\right)
$$

for any $q, 0<q<\infty$. Then $G_{2}(t ; q)$ is the only two-point cdf which has the mean 0 and the variance $\sigma^{2}$.

Lemma 5. The class $\mathcal{G}_{2}\left(0, \sigma^{2}\right)$ of two-point cdf's is represented with a parameter $q, 0<q<\infty$, as follows,

$$
\mathcal{G}_{2}\left(0, \sigma^{2}\right)=\left\{G_{2}(\cdot ; q) \mid 0<q<\infty\right\} \text {. }
$$

Let us define

$$
T_{\mathcal{F}}^{u}(x)=\sup _{F \in \mathcal{F}} T_{F}(x), T_{\mathcal{F}}^{\ell}(x)=\inf _{F \in \mathcal{F}} T_{F}(x)
$$

Then, from Lemma 1, we have the following lemma.

Lemma 6. Suppose $\mathcal{F}=\mathcal{F}(0)$ so that $\mu_{F}=0$ for all $F \in \mathcal{F}$, then $T_{\mathcal{F}}^{u}(x)$ and $T_{\mathcal{F}}^{\ell}(x)$ have the same property as $T_{F}(x)$ in Lemma 1 with $\mu_{F}$ replaced by 0 , except that $T_{\mathcal{F}}^{\ell}(x)$ is not always convex.

From above Lemma $6, T_{\mathcal{F}}^{u}(x)$ and $T_{\mathcal{F}}^{\ell}(x)$ have inverse functions $\left(T_{\mathcal{F}}^{u}\right)^{-1}(c)$ and $\left(T_{\mathcal{F}}^{\ell}\right)^{-1}(c)$ for all $c, c>0$, respectively. Thus we have shown the existence of the values of $\phi^{u}$ and $\phi^{*}$ :

$$
\begin{gathered}
\phi^{u}=\sup _{F \in \mathcal{F}}\left\{x \mid T_{F}(x)=c\right\}=\left(T_{\mathcal{F}}^{u}\right)^{-1}(c), \\
\phi^{*}=\inf _{F \in \mathcal{F}}\left\{x \mid T_{F}(x)=c\right\}=\left(T_{\mathcal{F}}^{\ell}\right)^{-1}(c) .
\end{gathered}
$$

\section{The Class $\mathcal{F}\left(\mu, \sigma^{2}\right)$}

In this section, we shall derive the maximal bound $\phi^{u}$ and the saddle value $\phi^{s}$ for $\phi(x, F)$ in the class $\mathcal{F}=\mathcal{F}\left(0, \sigma^{2}\right)$.

$$
\mathcal{F}\left(0, \sigma^{2}\right)=\left\{F \mid \int_{R} d F(t)=1, \int_{R} t d F(t)=0, \int_{R} t^{2} d F(t)=\sigma^{2}\right\}
$$

\subsection{The maximal bound $\phi^{u}$}

First, we prepare a useful proposition related to Schwartz inequalities. 
Proposition 3. (Feller [3, p.151]) If $F$ is an arbitrary $c d f$, then

$$
\left(\int_{A} u(t) v(t) d F(t)\right)^{2} \leq\left(\int_{A} u^{2}(t) d F(t)\right)\left(\int_{A} v^{2}(t) d F(t)\right)
$$

for any set $A$ and any functions $u, v$ for which the integrals on the right exist. Furthermore, the equality sign holds if and only if

$$
\int_{A}(a u(t)+b v(t))^{2} d F(t)=0 \text { for some } a, b \in R .
$$

Remark on Proposition 3. If $u$ and $v$ are linearly dependent, i.e., for some $a, b \in R, a u(t)+$ $b v(t)=0$, the condition (3.3) is satisfied for all $F \in \mathcal{F}$, and that if $u$ and $v$ are linearly independent, the condition (3.3) is satisfied only when the cdf $F$ is degenerated at one point in a set $A$.

We shall calculate $\phi^{u}$ and the maximizing point $\left(x^{u}, F^{u}\right)$ of the problem (2.9) by Proposition 3 .

$$
\begin{gathered}
\left(\int_{(x, \infty)}(t-x) d F(t)\right)^{2} \leq\left(\int_{(x, \infty)} d F(t)\right)\left(\int_{(x, \infty)}(t-x)^{2} d F(t)\right), \\
\left(\int_{(-\infty, x]}(t-x) d F(t)\right)^{2} \leq\left(\int_{(-\infty, x]} d F(t)\right)\left(\int_{(-\infty, x]}(t-x)^{2} d F(t)\right) .
\end{gathered}
$$

For all $F \in \mathcal{F}, T_{F}(x)=\int_{(x, \infty)}(t-x) d F(t)=c$ from $(2.9)$ and $\int_{R} d F(t)=1, \int_{R} t d F(t)=0$, $\int_{R} t^{2} d F(t)=\sigma^{2}$ from (3.1). Then, putting $p=\int_{(x, \infty)} d F(t)$, it holds that by adding (3.4) to $\left(3.4^{\prime}\right)$,

$$
\begin{gathered}
\frac{c^{2}}{p}+\frac{(x+c)^{2}}{1-p} \leq \sigma^{2}+x^{2}, 0<p<1, \\
-\frac{c}{p}-\sigma \sqrt{\frac{1-p}{p}} \leq x \leq-\frac{c}{p}+\sigma \sqrt{\frac{1-p}{p}}, 0<p<1 .
\end{gathered}
$$

Putting $q=\sqrt{(1-p) / p}, 0<q<\infty$, the upper bound of $x$ (the right-hand side of $(3.5))$ is represented by the parameter $q$.

(3.6) $\sup _{0<p<1}\left(\sigma \sqrt{\frac{1-p}{p}}-\frac{c}{p}\right)=\sup _{0<q<\infty}\left(\sigma q-c\left(1+q^{2}\right)\right)=\sup _{0<q<\infty}\left[-c\left(q-\frac{\sigma}{2 c}\right)^{2}+\frac{\sigma^{2}}{4 c}-c\right]=\frac{\sigma^{2}}{4 c}-c$,

where the supremum is attained at $q=\sigma / 2 c$ i.e., $p=4 c^{2} /\left(\sigma^{2}+4 c^{2}\right)$.

Then, we obtain the maximal bound $\phi^{u}$.

$$
\phi^{u}=\sup _{F \in \mathcal{F}}\left\{x \mid T_{F}(x)=c\right\}=\frac{\sigma^{2}}{4 c}-c .
$$

Since the equality holds in two Schwartz inequalities (3.4) and $\left(3.4^{\prime}\right)$, from the remark of Proposition 3, the maximizing cdf $F^{u}$ should be the two-point cdf. Then, we have

$$
F^{u}(t)=G_{2}\left(t ; \frac{\sigma}{2 c}\right)=\left(<-2 c, \frac{\sigma^{2}}{2 c}><\frac{\sigma^{2}}{\sigma^{2}+4 c^{2}}, \frac{4 c^{2}}{\sigma^{2}+4 c^{2}}>\right)
$$




$$
x^{u} \in \mathbf{x}^{u}=\mathbf{x}^{o}\left(F^{u}\right)=\left[-2 c, \frac{\sigma^{2}}{2 c}\right) \text {. }
$$

Theorem 1. For a class $\mathcal{F}\left(0, \sigma^{2}\right)$ of $c d f$ 's, the maximal bound $\phi^{u}$ is $\sigma^{2} / 4 c-c$ by (3.7) and all the maximizing points $\left(x^{u}, F^{u}\right) \in\left(\mathbf{x}^{u},\left\{F^{u}\right\}\right)$ are given by $F^{u}(t)=G_{2}(t ; \sigma / 2 c)$ in (3.8) and $\mathbf{x}^{u}=\left[-2 c, \sigma^{2} / 2 c\right)$ in $(3.9)$.

Remark on Theorem 1. From Lemma 2, the equation (3.9) means that the player 1 may decide a stopping level $x^{u}$ for some $x^{u} \in\left[-2 c, \sigma^{2} / 2 c\right)$ or $\sigma^{2} / 2 c-0$. If the player 1 decides any of the above stopping levels, he stops the process whenever $X_{n}=\sigma^{2} / 2 c$ is observed because the player 2 chooses only one cdf given by (3.8).

Corollary 1. For a class $\mathcal{F}\left(\mu, \sigma^{2}\right)$ of $c d f$ 's, the maximal bound $\phi^{u}$ is $\mu+\sigma^{2} / 4 c-c$ and all the maximizing points $\left(x^{u}, F^{u}\right) \in\left(\mathbf{x}^{u},\left\{F^{u}\right\}\right)$ are given by $F^{u}(t-\mu)=G_{2}(t ; \sigma / 2 c)$ and $\mathbf{x}^{u}=\left[\mu-2 c, \mu+\sigma^{2} / 2 c\right)$.

In the theory of extreme order statistics, by letting $Y=\max _{1 \leq n \leq \infty}\left[X_{n}-c n\right]$, it holds that $E\left[Y^{+}\right]<\infty$ if and only if $E\left[X^{+}\right]<\infty$ (See Pickands III [5, Cor. 2.2]). Corollary 1 also says that the maximal bound of $E\left[Y^{+}\right]$is calculated by using $\mu$ and $\sigma^{2}$ if the cdf $F$ is restricted in the class $\mathcal{F}\left(\mu, \sigma^{2}\right)$.

\subsection{The minimax value $\phi^{*}$}

Second, we shall calculate the minimax value $\phi^{*}$ of $(2.10)$ and the minimax-mizing point $\left(x^{*}, F^{*}\right) \in\left(\mathbf{x}^{*}, \mathcal{F}^{*}\right)$.

From Lemma $6, T_{\mathcal{F}}^{\ell}(x) \geq(-x)^{+}$for all $x \in R$. Then it holds that $T_{F^{*}}(x)=(-x)^{+} \leq T_{\mathcal{F}}^{\ell}(x)$ for $x \in(-\infty,-c]$ if a cdf $F^{*}$, which has all the mass on $[-c, \infty)$, is contained in $\mathcal{F}$. Since $T_{F^{*}}(x)=(-x)^{+}$is strictly decreasing on $(-\infty,-c]$, we have

$$
\phi^{*}=\inf _{F \in \mathcal{F}}\left\{x \mid T_{F}(x)=c\right\}=\left\{x \mid T_{F^{*}}(x)=c\right\}=-c .
$$

Such a class $\mathcal{F}^{*}$ of cdf's $F^{*}$ always exists in $\mathcal{F}$ for all $c, c>0$.

$$
\mathcal{F}^{*}=\left\{F \mid \int_{[-c, \infty)} d F(t)=1, F \in \mathcal{F}\right\}
$$

In particular, we can find the class $\mathcal{G}_{2}^{*}=\mathcal{G}_{2}^{*}\left(0, \sigma^{2}\right)$ of two-point cdf's in $\mathcal{F}^{*}$ from Lemma 5 .

$$
\mathcal{G}_{2}^{*}=\left\{G_{2}(\cdot ; q) \mid q \geq \frac{\sigma}{c}\right\} \text {. }
$$

It is easily shown that for any $F^{*} \in \mathcal{F}^{*}$ it is optimal for the player 1 to stop the process immediately. That is,

$$
\mathbf{x}^{*}=\mathbf{x}^{o}\left(F^{*}\right)=(-\infty,-c) \text { for all } F^{*} \in \mathcal{F} .
$$

Theorem 2. For a class $\mathcal{F}\left(0, \sigma^{2}\right)$ of $c d f$ 's, the minimax value $\phi^{*}$ is $-c$ by (3.10) and all the minimax-mizing points $\left(x^{*}, F^{*}\right) \in\left(\mathbf{x}^{*}, \mathcal{F}^{*}\right)$ are given by (3.11) and (3.12). In particular, there exists the class $\mathcal{G}_{2}^{*}$ of two-point cdf's in $\mathcal{F}^{*}$ by $\left(3.11^{\prime}\right)$.

Corollary 2. For a class $\mathcal{F}\left(\mu, \sigma^{2}\right)$ of $c d f$ 's, the minimax value $\phi^{*}$ is $\mu-c$, and all the minimaxmizing points $\left(x^{*}, F^{*}\right) \in\left(\mathbf{x}^{*}, \mathcal{F}^{*}\right)$ are given by

$$
\mathcal{F}^{*}=\left\{F \mid \int_{[\mu-c, \infty)} d F(t)=1, F \in \mathcal{F}\left(\mu, \sigma^{2}\right)\right\}, \mathbf{x}^{*}=(-\infty, \mu-c) .
$$




\subsection{The saddle value $\phi^{s}$}

Now, we shall derive the saddle value $\phi^{s}$ for $\phi(x, F)$ in $\mathcal{F}=\mathcal{F}\left(\mu, \sigma^{2}\right)$. We have a candidate $\left(\mathbf{x}^{*}, \mathcal{F}^{*}\right)$ for a set of saddle points $\left(\mathbf{x}^{s}, \mathcal{F}^{s}\right)$ to be checked by Proposition 2 . Since $\left(\mathbf{x}^{*}, \mathcal{F}^{*}\right)$ includes $\left(\mathbf{x}^{s}, \mathcal{F}^{s}\right)$ in general, we shall make an attempt to prove the inverse. From Theorem 2 it holds that $\phi(x, F)=-c$ for all $x \in \mathbf{x}^{*}$ and all $F \in \mathcal{F}^{*}$. Moreover, from (3.12), it holds that for any fixed $x \notin \mathbf{x}^{*}, \phi(x, F) \leq-c$ for all $F \in \mathcal{F}^{*}$. It suffices to prove for any fixed $F \in \mathcal{F}$,

$$
\phi(x, F) \geq-c \text { for all } x \in \mathbf{x}^{*} .
$$

Proof of (3.13). For any fixed $F$, it holds that $\phi(-\infty, F)=-c$ and $\phi(x, F)$ is a unimodal function of $x$ and maximized at the point $x^{\circ}(F)$ from the remark of $(1.1)$. When (i) $x^{\circ}(F) \geq-c$, $\phi(x, F) \geq-c$ for $x \in(-\infty,-c]$, i.e. (3.13) is true in the case (i). When (ii) $x^{o}(F) \leq-c$, $\phi(x, F) \geq \phi(-c, F)$ for $x \in\left[x^{o}(F),-c\right]$, so that (3.13) is true in the case (ii) if we can prove $\phi(-c, F) \geq-c$.

For $F \in \mathcal{F}, F$ has the mean 0 , so that $\bar{F}(-c)>0$ and

$$
\int_{(-c, \infty)} t d F(t)=-\int_{(-\infty,-c]} t d F(t) \geq c(1-\bar{F}(-c))
$$

Thus, we have from (1.1),

$$
\phi(-c, F)=\frac{\int_{(-c, \infty)} t d F(t)-c}{\bar{F}(-c)} \geq \frac{c(1-\bar{F}(-c))-c}{\bar{F}(-c)}=-c .
$$

This completes the proof.

Hence, we have the main theorem in this chapter.

Theorem 3. For a class $\mathcal{F}\left(0, \sigma^{2}\right)$ of cdf's, the saddle value $\phi^{s}$ is $-c$ and all the saddle points $\left(x^{s}, F^{s}\right) \in\left(\mathbf{x}^{s}, \mathcal{F}^{s}\right)$ are given by $\mathbf{x}^{s}=\mathbf{x}^{*}, \mathcal{F}^{s}=\mathcal{F}^{*}$ and $\mathcal{G}_{2}^{s}=\mathcal{G}_{2}^{*} \subset \mathcal{F}^{s}$ defined in Theorem 2.

Corollary 3. For a class $\mathcal{F}\left(\mu, \sigma^{2}\right)$ of $c d f$ 's, the saddle value $\phi^{s}$ is $\mu-c$ and all the saddle points $\left(x^{s}, F^{s}\right) \in\left(\mathbf{x}^{s}, \mathcal{F}^{s}\right)$ are given by $\mathbf{x}^{s}=\mathbf{x}^{*}, \mathcal{F}^{s}=\mathcal{F}^{*}$ defined in Corollary 2.

Theorem 3 says the class $\mathcal{F}\left(\mu, \sigma^{2}\right)$ is so rich for the player 2 that the player 1 must stop immediately. In this case, the information of the value $\sigma^{2}$ is useless for the player 1.

If we consider the problem in the class

$$
\mathcal{F}(\mu, \cdot, M)=\left\{F \mid \int_{A} d F(t)=1, \int_{A} t d F(t)=\mu, A=[\mu-M, \mu+M]\right\},
$$

the player 2 may choose the cdf which is degenerated at the point $\mu$ and the player 1 receives the reward of $\mu-c$, in which the information of the value $M$ is useless for the player 1 .

To remove the non-interesting strategies for the player 1 in the above two cases $\mathcal{F}\left(\mu, \sigma^{2}\right)$ and $\mathcal{F}(\mu, \cdot, M)$, we shall analyze the problem in the more restrictive class $\mathcal{F}\left(\mu, \sigma^{2}, M\right)$ of $(1.10)$ in the next section.

\section{The Class $\mathcal{F}\left(\mu, \sigma^{2}, M\right)$}

In this section, we shall derive the maximal bound $\phi^{u}$ and the saddle value $\phi^{s}$ in the more restrictive and interesting class $\mathcal{F}=\mathcal{F}\left(0, \sigma^{2}, M\right)$ (see (1.10)).

$$
\mathcal{F}\left(0, \sigma^{2}, M\right)=\left\{F \mid \int_{A} d F(t)=1, \int_{A} t d F(t)=0, \int_{A} t^{2} d F(t)=\sigma^{2}, A=[-M, M], \sigma \leq M\right\} .
$$


If $\sigma=M>0$, the class $\mathcal{F}$ contains only one cdf $G_{2}(t ; 1)=(<-M, M><1 / 2,1 / 2>)$. So, it is assumed that $\sigma<M$.

We calculate $\phi^{u}$ and the maximizing point $\left(x^{u}, F^{u}\right)$ in the same fashion as (3.6) with the additional constraint $\sigma / M \leq q \leq M / \sigma$,

$$
\phi^{u}=\max _{\sigma / M \leq q \leq M / \sigma}\left[\sigma q-c\left(1+q^{2}\right)\right] .
$$

Theorem 4. For a class $\mathcal{F}\left(0, \sigma^{2}, M\right)$ of cdf's, $\sigma<M$, the maximal bound $\phi^{u}$ and all the maximizing points $\left(x^{u}, F^{u}\right) \in\left(\mathbf{x}^{u}, \mathcal{F}^{u}\right)$ are as follows:

(i) When $0 \leq c \leq \sigma^{2} / 2 M$,

$$
\begin{gathered}
\phi^{u}=M-c\left(1+\frac{M^{2}}{\sigma^{2}}\right), \mathbf{x}^{u}=\left[-\frac{\sigma^{2}}{M}, M\right) \\
F^{u}(t)=G_{2}\left(t ; \frac{M}{\sigma}\right)=\left(<-\frac{\sigma^{2}}{M}, M><\frac{M^{2}}{\sigma^{2}+M^{2}}, \frac{\sigma^{2}}{\sigma^{2}+M^{2}}>\right) .
\end{gathered}
$$

(ii) When $\sigma^{2} / 2 M \leq c \leq M / 2$, the same result as Theorem 1 holds, i.e.,

$$
\begin{gathered}
\phi^{u}=\frac{\sigma^{2}}{4 c}-c, \mathbf{x}^{u}=\left[-2 c, \frac{\sigma^{2}}{2 c}\right), \\
F^{u}(t)=G_{2}\left(t ; \frac{\sigma}{2 c}\right)=\left(<-2 c, \frac{\sigma^{2}}{2 c}><\frac{\sigma^{2}}{\sigma^{2}+4 c^{2}}, \frac{4 c^{2}}{\sigma^{2}+4 c^{2}}>\right) .
\end{gathered}
$$

(iii) When $M / 2 \leq c \leq M$,

$$
\begin{gathered}
\phi^{u}=\frac{\sigma^{2}}{M}-c\left(1+\frac{\sigma^{2}}{M^{2}}\right), \mathbf{x}^{u}=\left[-M, \frac{\sigma^{2}}{M}\right) \\
F^{u}(t)=G_{2}\left(t ; \frac{\sigma}{M}\right)=\left(<-M, \frac{\sigma^{2}}{M}><\frac{\sigma^{2}}{\sigma^{2}+M^{2}}, \frac{M^{2}}{\sigma^{2}+M^{2}}>\right) .
\end{gathered}
$$

Remark on Theorem 4. When $c>M$, i.e. the observation cost is larger than the maximal value of the observation, it is natural for the player 1 to stop immediately, i.e., $\phi^{u}=-c, \mathcal{F}^{u}=\mathcal{F}$ and $\mathbf{x}^{u}=(-\infty,-M)$. The shape of $\phi^{u}$ as the function of $c$ is illustrated in Figure 1 at the end of this paper. Furthermore, if the results for the class $\mathcal{F}\left(\mu, \sigma^{2}, M\right)$ are required, Lemma 2 is useful for the theorems in this section.

Now, we shall derive the saddle value $\phi^{s}$. By calculating $T_{F}(x)$ of $(1.2)$ for the cdf's $G_{2}(\cdot ; M / \sigma)$ and $G_{2}(\cdot ; \sigma / M)$, we have, from $(2.8)$ and Lemma 6 ,

$$
\begin{gathered}
T_{\mathcal{F}}^{\ell}(x) \geq(-x)^{+}=-x=T_{G_{2}(\cdot ; M / \sigma)}(x) \text { for } x \in\left[-M,-\sigma^{2} / M\right], \\
T_{\mathcal{F}}^{\ell}(x) \geq(-x)^{+}=0=T_{G_{2}(\cdot ; \sigma / M)}(x) \text { for } x \in\left[\sigma^{2} / M, M\right] .
\end{gathered}
$$

If the observation cost $c$ is a constant such that $M \geq c \geq \sigma^{2} / M$, the equality $\phi^{*}=\left(T_{\mathcal{F}}^{\ell}\right)^{-1}(c)=$ $-c$ is satisfied from (4.2). In this case, the saddle value $\phi^{s}$ and the saddle point $\left(x^{s}, F^{s}\right)$ are the same ones given in Theorem 3 , because the player 2 can choose a cdf $G_{2}(\cdot, M / \sigma)$ in this restrictive class $\mathcal{F}=\mathcal{F}\left(0, \sigma^{2}, M\right)$, i.e., $G_{2}(\cdot ; M / \sigma) \in \mathcal{F}\left(0, \sigma^{2}, M\right) \subset \mathcal{F}\left(0, \sigma^{2}\right)$. So that we confine our consideration to the case:

$$
0<c<\sigma^{2} / M
$$


On the other hand, from (4.2) and (4.3), it holds that

$$
\begin{gathered}
\inf _{F \in \mathcal{F}} \phi(x, F) \leq \phi\left(x, G_{2}(\cdot ; M / \sigma)\right)=-c \text { for } x \in\left[-M,-\sigma^{2} / M\right), \\
\inf _{F \in \mathcal{F}} \phi(x, F) \leq \phi\left(x, G_{2}(\cdot ; \sigma / M)\right)=-\infty \text { for } x \in\left[\sigma^{2} / M, M\right],
\end{gathered}
$$

because the player 1 stops immediately in the case of $\left(4.2^{\prime}\right)$ or he cannot stop in the case of $\left(4.3^{\prime}\right)$. Then, the player 1 must decide his stopping level $x$ in the interval

$$
\mathbf{x}^{M} \stackrel{\text { def }}{=}\left[-\frac{\sigma^{2}}{M}, \frac{\sigma^{2}}{M}\right)
$$

in order not to make his reward $\inf _{F \in \mathcal{F}} \phi(x, F) \leq-c$, where $-c$ is the reward of immediately stopping or the saddle value $\phi^{s}=-c$ in Section 3 .

Hereafter, we consider the stopping problem $\phi(x, F)$ under the restriction of (4.4) and (4.5). To begin with, we prepare the following three lemmas.

Lemma 7. If $F$ is an arbitrary cdf defined on a finite interval $[a, b]$ with the mean $\mu(a \leq \mu \leq$ $b, a<b)$, then

$$
\int_{[a, b]}(t-\mu)^{2} d F(t) \leq(\mu-a)(b-a) .
$$

Furthermore, the equality holds if and only if

$$
F=\left(<a, b><\frac{b-\mu}{b-a}, \frac{\mu-a}{b-a}>\right) .
$$

Proof. Since $f(t)=(t-\mu)^{2}$ is strictly convex, we have

$$
\int_{[a, b]}(t-\mu)^{2} d F(t) \leq(a-\mu)^{2} \int_{[a, \mu)} d F(t)+(b-\mu)^{2} \int_{(\mu, b]} d F(t) .
$$

The equality holds iff $F$ satisfies $\int_{[a, \mu)} d F(t)=\int_{\{a\}} d F(t)$ and $\int_{(\mu, b]} d F(t)=\int_{\{b\}} d F(t)$. Such a cdf is uniquely determined by (4.6). This completes the proof.

Lemma 8. For any strategy $(x, F) \in\left(\mathrm{x}^{M}, \mathcal{F}\right)$, if $F$ has a positive probability on the interval $(x, M)$ and satisfies $\phi(x, F) \geq-c$, then there exists a cdf $F^{\prime \prime} \in \mathcal{F}$ such that $F^{\prime \prime}$ has no probability on the interval $(x+\varepsilon, M)$ for an arbitrary small $\varepsilon>0$, and it satisfies $\phi\left(x, F^{\prime \prime}\right) \leq \phi(x, F)$.

Proof. Let $x^{\prime}=x+\varepsilon, p=\int_{\left(x^{\prime}, M\right)} d F(t)>0$ and $y=p^{-1} \int_{\left(x^{\prime}, M\right)} t d F(t), x^{\prime}<y<M$. From Lemma 7 , the probability $p$ of $F$ is allotted to the points $x^{\prime}$ and $M$ without changing its mean 0 . Let a cdf $F^{\prime}$ be

$$
F^{\prime}(t)=\left\{\begin{array}{cl}
F(t) & , \quad t<x^{\prime} \\
F\left(x^{\prime}\right)+\left(<x^{\prime}, M><p \frac{M-y}{M-x^{\prime}}, p \frac{y-x^{\prime}}{M-x^{\prime}}>\right) & , \quad x^{\prime} \leq t<M \\
1 & , \quad M \leq t
\end{array}\right.
$$

which has the variance greater than $\sigma^{2}$. The difference $\gamma^{2}$ of the variances is given by

$$
\begin{aligned}
\gamma^{2} & =\int_{A} t^{2} d F^{\prime}(t)-\int_{A} t^{2} d F(t) \\
& =p\left[\left(x^{\prime}\right)^{2} \frac{M-y}{M-x^{\prime}}+M^{2} \frac{y-x^{\prime}}{M-x^{\prime}}\right]-\int_{\left(x^{\prime}, M\right)} t^{2} d F(t)>0 .
\end{aligned}
$$


Putting $\lambda=\sigma^{2} /\left(\sigma^{2}+\gamma^{2}\right)$, let us define

$$
F^{\prime \prime}(t)=\lambda F^{\prime}(t)+(1-\lambda) G_{1}(t), 0<\lambda<1,
$$

where $G_{1}$ is the cdf degenerated at the origin 0 . Then we find $F^{\prime \prime}$ is in $\mathcal{F}$, i.e., $F^{\prime \prime}$ has the mean 0 and the variance $\sigma^{2}$. From Lemma 4 and (4.8) it holds that

$$
\phi\left(x, F^{\prime \prime}\right)=\frac{\lambda \bar{F}^{\prime}(x)}{\bar{F}^{\prime \prime}(x)} \phi\left(x, F^{\prime}\right)+\frac{(1-\lambda) \bar{G}_{1}(x)}{\bar{F}^{\prime \prime}(x)} \phi\left(x, G_{1}\right),
$$

where $\bar{G}_{1}(x)=1$ and $\phi\left(x, G_{1}\right)=-c$ for $x<0, \bar{G}_{1}(x)=0$ for $x \geq 0$. Since $\bar{F}^{\prime}(x)=\bar{F}(x)$ and $\phi\left(x, F^{\prime}\right)=\phi(x, F)$ from (4.7) and (1.1), we have $\phi\left(x, F^{\prime \prime}\right) \leq \phi(x, F)$ because $\phi\left(x, F^{\prime \prime}\right)$ in (4.9) is a convex combination of $\phi(x, F)(\geq-c)$ and $-c$.

Now, we shall redefine the cdf $F^{\prime \prime}$ which has no probability on the interval $\left(x^{\prime}, M\right)$.

When $x \geq 0, F^{\prime \prime}$ in (4.8) has no probability on $\left(x^{\prime}, M\right)$ and the result holds. When $x<0$, let $x^{\prime}=x+\varepsilon<0$ and $F_{(1)}^{\prime \prime} \stackrel{\text { def }}{=} F^{\prime \prime}$ in $(4.8)$ has a probability mass $p_{(1)}=F_{(1)}^{\prime \prime}(0)-F_{(1)}^{\prime \prime}(0-0)$ at the origin 0 on $\left(x^{\prime}, M\right)$. If the mass $p_{(1)}$ of $F_{(1)}^{\prime \prime}$ is allotted to the points $x^{\prime}$ and $M$, then we can define $F_{(2)}^{\prime}$ and $F_{(2)}^{\prime \prime}$ similarly as (4.7) and (4.8):

$$
\begin{aligned}
& F_{(2)}^{\prime}(t)=F_{(1)}^{\prime \prime}(t)+\left(<x^{\prime}, 0, M><p_{(1)} \frac{M}{M+\left|x^{\prime}\right|},-p_{(1)}, p_{(1)} \frac{\left|x^{\prime}\right|}{M+\left|x^{\prime}\right|}>\right) \\
& F_{(2)}^{\prime \prime}(t)=\lambda_{(2)} F_{(2)}^{\prime}(t)+\left(1-\lambda_{(2)}\right) G_{1}(t), \text { where } \lambda_{(2)}=\frac{\sigma^{2}}{\sigma^{2}+p_{(1)} M\left|x^{\prime}\right|}
\end{aligned}
$$

Let us define $F_{(n+1)}^{\prime} \in \mathcal{F}$ and $F_{(n+1)}^{\prime \prime} \in \mathcal{F}$ recursively by $\left(4.7^{\prime}\right)$ and $\left(4.8^{\prime}\right)$ with the subindexes (2) and (1) replaced by $(n+1)$ and $(n)$ respectively $(n \geq 1)$. The mass $p_{(n+1)}$ of $F_{n+1}^{\prime \prime}$ is given successively by

$$
p_{(n+1)}=\frac{p_{(n)}}{k+p_{(n)}}, k=\frac{\sigma^{2}}{M\left|x^{\prime}\right|}>0, n \geq 1
$$

where $\lim _{n \rightarrow \infty} p_{(n)}=0$ for any $p_{(1)}$. By the similar argument about $F^{\prime \prime}=F_{(1)}^{\prime \prime}$ in $(4.9), F_{(n)}^{\prime \prime} \in$ $\mathcal{F}, n \geq 1, F^{\prime \prime} \stackrel{\text { def }}{=} \lim _{n \rightarrow \infty} F_{(n)}^{\prime \prime} \in \mathcal{F}$ and $\phi\left(x, F^{\prime \prime}\right) \leq \phi\left(x, F_{(n)}^{\prime \prime}\right) \leq \phi(x, F), n \geq 1$. This completes the proof.

Lemma 9. For any strategy $(x, F) \in\left(\mathbf{x}^{M}, \mathcal{F}\right)$, if $F$ has a positive probability on the interval $(-M, x)$ and it satisfies $\phi(x, F) \geq-c$, then there exists a cdf $F^{\prime \prime} \in \mathcal{F}$ such that $F^{\prime \prime}$ has no probability on the interval $(-M, x)$, and it satisfies $\phi\left(x, F^{\prime \prime}\right) \leq \phi(x, F)$.

Proof. Let $p=\int_{(-M, x)} d F(t)>0$ and $y=p^{-1} \int_{(-M, x)} t d F(t),-M<y<x$. From Lemma 7 , the probability $p$ of $F$ is allotted to the point $-M$ and $x$ without changing its mean 0 . Let a cdf $F^{\prime}$ be

$$
F^{\prime}(t)=\left\{\begin{array}{cl}
F(-M)+\left(<-M, x><p \frac{x-y}{M+x}, p \frac{M+y}{M+x}>\right) & , \quad-M \leq t \leq x \\
F(t) & , \quad x<t
\end{array}\right.
$$

which has the variance greater than $\sigma^{2}$. The difference $\gamma^{2}$ of the variances is given by

$$
\gamma^{2}=p\left[x^{2} \frac{M+y}{M+x}+M^{2} \frac{x-y}{M+x}\right]-\int_{(-M, x)} t^{2} d F(t)>0 .
$$


Putting $\lambda=\sigma^{2} /\left(\sigma^{2}+\gamma^{2}\right)$, let a cdf $F^{\prime \prime}$ be defined similarly by (4.8). Then, in the same fashion as in Lemma 8 , the result holds.

Let us define for any $x \in\left[-\sigma^{2} / M, \sigma^{2} / M\right]$, a three-point $\operatorname{cdf} G_{3}^{M}(\cdot ; x) \in \mathcal{F}$ which has all the mass at three points $-M, x, M$ with the mean 0 and the variance $\sigma^{2}$. This cdf is uniquely determined by

$$
G_{3}^{M}(t ; x)=\left(<-M, x, M><\frac{M x+\sigma^{2}}{2 M(M+x)}, \frac{M^{2}-\sigma^{2}}{M^{2}-x^{2}}, \frac{\sigma^{2}-M x}{2 M(M-x)}>\right),
$$

and let $\mathcal{G}_{3}^{M}=\left\{G_{3}^{M}(t ; x) \mid-\sigma^{2} / M \leq x \leq \sigma^{2} / M\right\}$. Note that if $x=\sigma^{2} / M$ or $-\sigma^{2} / M, G_{3}^{M}(t ; x)$ becomes the two-point cdf $G_{2}(t ; \sigma / M)$ or $G_{2}(t ; M / \sigma)$ respectively.

Now, we shall derive a saddle point. It holds, from Theorem 3 , that

$$
-c=\sup _{x \in R} \inf _{F \in \mathcal{F}\left(0, \sigma^{2}\right)} \phi(x, F) \leq \sup _{x \in R} \inf _{F \in \mathcal{F}} \phi(x, F) .
$$

Then the player 1 would decide a stopping level $x$ in the following set

$$
\{x \mid \phi(x, F) \geq-c \text { for all } F \in \mathcal{F}\} \cap \mathbf{x}^{M} \stackrel{\text { def }}{=} \mathbf{x}_{c}^{M} .
$$

This set is not empty because $x=-c$ is contained in it.

Remark on Lemmas 8 and 9. Lemmas mean the following : for any $x \in \mathbf{x}_{c}^{M}$, if the player 1 (he) decides his stopping level $x$, the player 2(she) may choose her cdf which has no probability on the interval $(-M, x) \cup(x+\varepsilon, M)$ for an arbitrary small $\varepsilon>0$, where her cdf converges to $G_{3}^{M}(\cdot ; x)$ of $(4.11)$ as $\varepsilon$ goes to 0 . In this situation, his stopping set becomes $\{X \in(x, x+\varepsilon] \cup\{M\}\}$ for an arbitrary small $\varepsilon>0$, i.e., $\{X \in\{M\}\}$ or $\{X \in\{x\} \cup\{M\}\}$.

From the above remark, we have for any $x \in \mathbf{x}_{c}^{M}$,

$$
\inf _{F \in \mathcal{F}} \phi(x, F)=\min \left[\phi\left(x, G_{3}^{M}(\cdot ; x), \phi\left(x-0, G_{3}^{M}(\cdot ; x)\right)\right]\right.
$$

If there exists a point $x^{s} \in \mathbf{x}_{c}^{M}$ such that

$$
\phi\left(x^{s}, G_{3}^{M}\left(\cdot ; x^{s}\right)\right)=\left(T_{\mathcal{G}_{3}^{M}}^{\ell}\right)^{-1}(c)\left(=\phi\left(x^{s}-0, G_{3}^{M}\left(\cdot ; x^{s}\right)\right)\right) \geq-c
$$

the strategy $\left(x^{s}, G_{3}^{M}\left(\cdot ; x^{s}\right)\right), x \in \mathrm{x}_{c}^{M}, G_{3}^{M}\left(\cdot ; x^{s}\right) \in \mathcal{G}_{3}^{M} \subset \mathcal{F}$, is the saddle point and $\phi^{s}=$ $\left(T_{\mathcal{G}_{3}{ }^{M}}\right)^{-1}(c)$ is the saddle value. Because, from (4.14) and (2.10), the following relation is satisfied.

$$
\begin{gathered}
\min \left[\phi\left(x^{s}, G_{3}^{M}\left(\cdot ; x^{s}\right), \phi\left(x^{s}-0, G_{3}^{M}\left(\cdot ; x^{\varsigma} s\right)\right)\right] \leq \sup _{x \in \mathbf{x}_{c}^{M}} \inf _{F \in \mathcal{F}} \phi(x, F)\right. \\
\leq \inf _{F \in \mathcal{F}} \sup _{x \in \mathbf{x}_{c}^{M}} \phi(x, F) \leq \inf _{F \in \mathcal{G}_{3}^{M}} \sup _{x \in \mathbf{x}_{c}^{M}} \phi(x, F)=\left(T_{\mathcal{G}_{3}^{M}}^{\ell}\right)^{-1}(c)=\phi\left(x^{s}, G_{3}^{M}\left(\cdot ; x^{s}\right)\right) .
\end{gathered}
$$

For any $x, y \in\left[-\sigma^{2} / M, \sigma^{2} / M\right]$, it is shown, by the graph of $T_{G_{3}^{M}(\cdot ; y)}(x)$, that

$$
T_{G_{3}^{M}(\cdot ; y)}(x)=\int_{x}^{M}\left[1-G_{3}^{M}(t ; y)\right] d t \geq T_{G_{3}^{M}(\cdot ; x)}(x)=\int_{x}^{M}\left[1-G_{3}^{M}(t ; x)\right] d t=\frac{\sigma^{2}-M x}{2 M} .
$$

This line segment $T=\left(\sigma^{2}-M x\right) / 2 M$ connects the points $\left(-\sigma^{2} / M, \sigma^{2} / M\right)$ and $\left(\sigma^{2} / M, 0\right)$ in $(x, T)$-plane ,and the equality in (4.16) holds only when $y=x$. 
Then, we have proved (4.15).

$$
\begin{gathered}
\left(T_{G_{3}^{M}}^{\ell}\right)^{-1}(c)=\frac{\sigma^{2}}{M}-2 c=\left(\frac{\sigma^{2}}{M}-c\right)^{+}-c \geq-c, \\
x^{s}=\left(\frac{\sigma^{2}}{M}-c\right)^{+}-c, F^{s}=G_{3}^{M}\left(\cdot ; x^{s}\right) .
\end{gathered}
$$

Hence, we have the main theorem of our problem.

Theorem 5. For a class $\mathcal{F}\left(0, \sigma^{2}, M\right)$ of $c d f$ 's, $\sigma \leq M$, we have the following solution:

(i) When $\sigma^{2} / M \leq c \leq M$, the same result as Theorem 3 holds, that is, the saddle value $\phi^{s}$ and all the saddle points $\left(x^{s}, F^{s}\right) \in\left(\mathbf{x}^{s}, \mathcal{F}^{s}\right)$ are given by

$$
\phi^{s}=-c, \mathbf{x}^{s}=[-M,-c] \text { and } \mathcal{F}^{s}=\left\{F \mid \int_{[-c, M]} d F(t)=1, F \in \mathcal{F}\left(0, \sigma^{2}, M\right)\right\}
$$

(ii) When $0<c<\sigma^{2} / M$, the saddle value $\phi^{s}$ and the saddle point $\left(x^{s}, F^{s}\right)$ are given by

$$
\phi^{s}=\left(\sigma^{2} / M-c\right)^{+}-c, x^{s}=\left(\sigma^{2} / M-c\right)^{+}-c \text { and } F^{s}(t)=G_{3}^{M}\left(t ; x^{s}\right) \text { defined by (4.11). }
$$

Unfortunately, we could not show the uniqueness of the saddle point $\left(x^{s}, F^{s}\right)$ in Case (ii). Finally, we conclude this paper by illustrating $\phi^{u}$ of Theorem 4 and $\phi^{s}$ of Theorem 5 in Figure 1.

Acknowledgments The author would like to thank two anonymous referees for their helpful comments.

\section{References}

[1] Chow,Y.S., H.Robbins and D.Siegmund, Great Expectations : The Theory of Optimal Stopping, Houghton-Mifflin, New York, 1971.

[2] Ekeland,I. and R.Teman, Convex Analysis and Variational Problems, North-Holland, 1976.

[3] Feller,W., An Introduction to Probability Theory and its Applications, Vol. II, Wiley, New York, 1968.

[4] Nakagami,J. and M.Yasuda, "Saddle Point of an Inventory Problem", J. Information and Optimization Sciences, 2 (1981), pp. 181-191.

[5] Pickands,J.III, "Extreme Order Statistics with Cost of Sampling”, Adv. Appl. Prob., 15 (1983), pp. 783-797.

Jun-ichi NAKAGAMI

Department of Mathematics

Faculty of Science, Chiba University 1-33 Yayoi-cho, Chiba-city, 260 JAPAN 


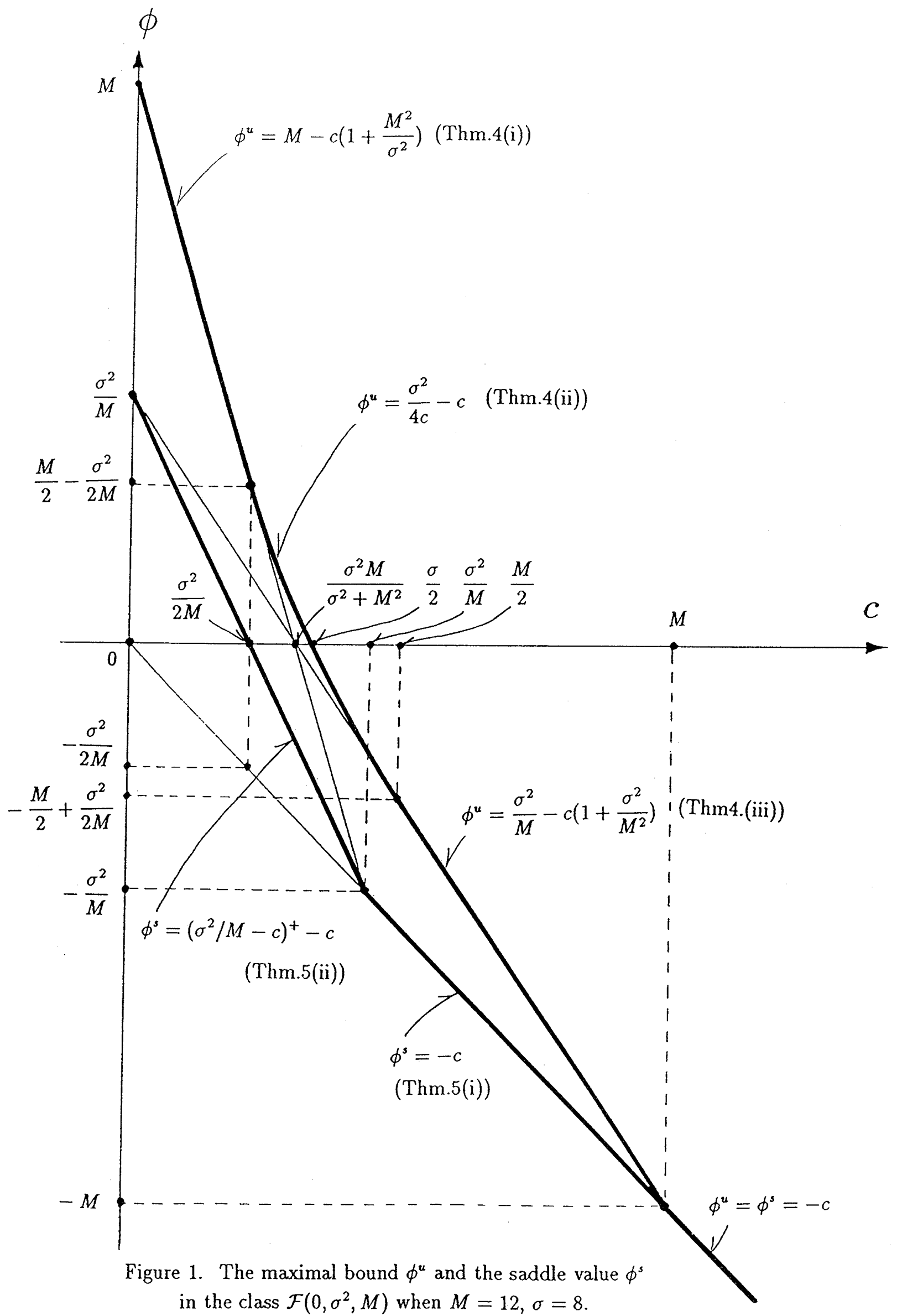

